\title{
Impact of daily yoga-based exercise on pain, catastrophizing, and sleep amongst individuals with fibromyalgia
}

\author{
This article was published in the following Dove Press journal: \\ Journal of Pain Research
}

\author{
Asimina Lazaridou' \\ Alexandra Koulouris' \\ Jaime K Devine ${ }^{2}$ \\ Monika Haack ${ }^{2}$ \\ Robert N Jamison (D) \\ Robert R Edwards' \\ Kristin L Schreiber (D) \\ 'Department of Anesthesiology, \\ Perioperative, and Pain Medicine, \\ Brigham and Women's Hospital, Harvard \\ Medical School, Chestnut Hill, MA 02467, \\ USA; ${ }^{2}$ Department of Neurology, \\ Harvard Medical School, Beth Israel \\ Deaconess Medical Center, Boston, MA \\ 022I5, USA
}

Background: Fibromyalgia (FM) is a chronic widespread pain disorder characterized by negative affect, sleep disturbance, and fatigue. This uncontrolled pilot study investigated the efficacy of daily yoga-based exercise to improve FM symptoms and explored baseline phenotypic characteristics associated with the greatest benefit.

Methods: FM patients ( $\mathrm{n}=46$, with 36 completers) reported psychosocial functioning and a range of FM symptoms using validated instruments before and after participation in Satyananda yoga, which included weekly in-person pain-tailored group classes for 6 weeks and daily home yoga video practice.

Results: Changes in FM symptoms from pre- to post-yoga were variable amongst participants. Group means for pain decreased, as reported by average daily diary and Brief Pain Inventory, with greater home practice minutes associated with a greater decrease in pain. Average daily ratings of sleep and fatigue improved. Pain catastrophizing was decreased overall, with greater change correlated to a decrease in FM symptoms. We did not observe any group mean changes in actigraphy sleep efficiency, Patient-Reported Outcomes Measurement Information System-anxiety and the Revised Fibromyalgia Impact Questionnaire. Multilevel Modeling analysis revealed a significant interaction between anxiety and catastrophizing for end-study sleep efficiency, fatigue, and pain, such that patients with higher baseline catastrophizing and lower baseline anxiety reported less pain and fatigue, and higher sleep efficiency after the sixth week of yoga practice.

Conclusion: This pilot study suggests that yoga may reduce pain and catastrophizing, as well as improve sleep, but these changes were modest across study participants. Greater uptake of home yoga practice as well as a phenotype of higher baseline catastrophizing combined with lower baseline anxiety were associated with greater impact. Future randomized, controlled trials comparing different types of yoga or exercise will allow determination of the most effective treatments for FM and allow closer targeting to the patients who will benefit most from them.

Keywords: yoga, pain, fibromyalgia, sleep, catastrophizing

\section{Introduction}

Fibromyalgia (FM), characterized by persistent, widespread pain, myofascial tenderness, negative affect, and dysregulated sleep, affects an estimated 5 million adults in the US ( $2 \%$ prevalence in 2005 ), though recent population-based surveys have estimated higher rates. ${ }^{1,2}$ FM involves a complex and diverse set of symptoms and proposed biopsychosocial mechanisms including 1) alterations in central pain-modulatory processes in the spinal cord and brain, 2) a prominent role of negative affective factors in
Correspondence: Kristin L Schreiber Department of Anesthesiology, Perioperative, and Pain Medicine, Brigham and Women's Hospital, Harvard Medical School, 75 Francis Street, Boston, MA 02115 , USA

Tel + 6177328218

Email klschreiber@bwh.harvard.edu 
maintaining pain and disability, 3) disrupted sleep and circadian rhythms and related fatigue, and 4) a relative lack of efficacy of many pharmacologic treatments. ${ }^{2}$

Psychosocial functioning plays a key role in shaping individual differences in pain. Negative affect and catastrophizing amplify pain sensitivity and influence pain modulation in many chronic pain conditions, including FM, as well as low back pain, osteoarthritis, and rheumatoid arthritis. ${ }^{3,4}$ Interestingly, exercise has been shown to impact a broad array of FM symptoms, ${ }^{5}$ with collective evidence suggesting that moderately intense, relatively frequent (at least $3 \times /$ week) and sustained (4-6 weeks) exercise may reduce not only pain and fatigue, but also depression symptoms. ${ }^{6}$ However, finding an exercise routine that is 1) gentle enough not to trigger the new onset of pain, soreness, and thereby, catastrophizing about pain, 2) adaptable to the individual, and 3) habitually sustainable over the long term, is a substantial challenge. Yoga, with its holistic approach, may be well-adapted to meet these criteria, and has shown promise in reducing pain and functional outcomes in both low back and FM pain. ${ }^{1,7-14}$ Most patients with FM also suffer from significant disturbances initiating or maintaining sleep, ${ }^{15}$ and robust relationships are observed between pain severity and sleep disturbance in FM patients, ${ }^{16}$ suggesting this to be an important aim for treatment strategy success.

The aim of this uncontrolled pilot study was to assess the effectiveness daily yoga-based exercise, consisting of a specially designed gentle 6-week program to reduce FM symptoms, including pain and sleep disturbance, and with a focus on the role of negative cognitions about pain such as catastrophizing.

\section{Materials and methods}

\section{Setting, participants, and study design}

The Partners Institutional Review Board approved this study, which was a pilot uncontrolled trial without a control comparison group. Participants were recruited using physical flyers and a centralized clinical data registry. Inclusion criteria included; 1) 18-75 years of age, 2) chronic pain with a diagnosis of FM (Wolfe et al 2011 criteria) for $>6$ months' duration, 3) stable medications prior to entering the study, 4) average pain score $\geq 4 / 10$, 5) sleep disturbance, defined as Pittsburgh Sleep Quality Index (PSQI) score $\geq 5,6$ ) able to speak and understand English, 7) access to a computer/tablet/smartphone at home, 8) and willing and physically able to participate in yoga-based exercise. Exclusion criteria included; 1) ongoing cancer treatment, 2) acute osteomyelitis/bone disease, 3) chronic systemic inflammatory disease, 4) schizophrenia, delusional disorder, psychotic disorder, or dissociative disorder judged to interfere with study participation, 5) pregnancy, 6) unstable systemic illness or injury requiring urgent surgery or treatment, 7) active addiction disorder, 8) current rigorous daily exercise routine ( $>20 \mathrm{mins} /$ day and $>5 \times /$ week), and 9) current regular meditative practice (e.g tai-chi, meditative form of yoga or contemplative prayer) for $>20 \mathrm{~min} /$ week. Informed consent procedures were performed in accordance with the Declaration of Helsinki for all participants. Patients who dropped out before week 6 or attended $<4 / 6$ weekly yoga classes were not included in the analysis.

\section{Yoga study procedures}

Participants underwent initial telephone screening, and eligible participants who gave consent were invited for a baseline research visit followed by a 6-week yoga program. Once a sufficient number of participants to fill a class were enrolled (group sizes of $\mathrm{n} \sim 10$ ), time of day and day of the week for classes was determined based on participants' and instructor's collective schedules. The study included 5 separate consecutive yoga cycles/groups, with 6-10 participants completing the yoga program per cycle, which took place at a satellite outpatient clinical and research center connected to a large tertiary referral academic hospital.

\section{Satyananda Yoga program}

Subjects participated in a yoga program specifically adjusted for chronic pain patients, led by a certified yoga instructor and doctoral-level psychologist. Each week, there was an in-person session that lasted $1.5 \mathrm{hrs}$ and included asanas, meditation, and other mindfulness-based practices (see Appendix). Satyananda Yoga is a traditional form of yoga which includes asanas (physical exercises), pranayama (breathing exercises), pratyahara (mind focusing practices), and meditation (breathing awareness, awareness of senses, awareness of thoughts, and yoga nidra); it is gentle and may be adapted to be less physically demanding, ${ }^{17,18}$ integrating development of physical, mental, emotional, psychic, and spiritual aspects of one's being. In order to encourage the integration of these practices into everyday life, participants were also instructed to follow a daily 30-min yoga video while at home in between in-person classes with the instructor. These videos featured the same instructor as in-person and consisted of a condensed 
combination of meditation, breathing exercises, and asanas, which had been introduced in the class that week (i.e., 6 different videos sent throughout the program, a new one for each week).

\section{Sociodemographic data and psychosocial characteristics}

Sociodemographic information was collected including age, race, education, and current occupational status. Only female participants responded to study advertisements. Data were collected during the baseline assessment week and the 6th week of yoga intervention (end of study intervention), via the secure REDCap survey system email link.

\section{Pain Catastrophizing}

The Pain Catastrophizing Scale (PCS), ${ }^{19}$ which has been widely used in chronic pain patients and controls, ${ }^{20,21}$ was used to measure catastrophic thinking (rumination, magnification, and helplessness) about pain.

\section{Emotional Distress-Anxiety and Depression}

The Patient-Reported Outcomes Measurement Information System (PROMIS) anxiety (7 items) and depression (8 items) short forms, which are widely used and extensively validated, ${ }^{22,23}$ were used.

\section{Fibromyalgia symptoms}

In order to measure general FM symptomatology, the Revised Fibromyalgia Impact Questionnaire (FIQR), ${ }^{24}$ a 21-item assessment of functioning and overall impact of FM symptoms, was completed.

\section{Self-Reported Sleep Assessment}

The Pittsburgh Sleep Quality Inventory (PSQI), which includes questions regarding sleep quality, latency, duration, disturbances, habitual sleep efficiency, daytime functionality due to sleep, and the use of sleep medications, ${ }^{25}$ was used, with score $\geq 5$ considered "poor sleep quality".

\section{Sleep Actigraphy}

A wrist actigraph (Actiwatch 2) was worn by participants during the baseline assessment week and during the 6th week of the yoga program in order to estimate sleep efficiency. Activity count data was downloaded and habitual sleep parameters determined using Philips Actiware default settings for sleep-wake determination. Average sleep efficiency for a week was calculated as: (sleep time/in bed time) $\times 100$, averaged over 6 nights.
Pain

Participants completed the Brief Pain Inventory (BPI), ${ }^{26}$ including 4 pain intensity questions (pain now, average pain, worst pain, least pain) rated on a 0 to 10 scale.

\section{Ecological momentary assessment (daily diaries)}

During the baseline assessment week and the 6th week of yoga intervention, patients completed 7 days of electronic diary entries at home, sent via the secure REDCAP survey system email link each morning and evening. This included pain ratings, anxiety, fatigue, stress, and subjective sleep quality.

\section{Yoga practice}

At the end of each week of the yoga program (i.e., on the day before each yoga class), participants completed a "weekly check-in" survey, reporting the number of days and amount of time/number of days that they practiced yoga at home since the last yoga class (over a 5-day period).

\section{Data analysis}

A total of 46 participants were enrolled, with 36 participants completing the study (Appendix- study flow diagram). Those who dropped out before week 6 or attended $<4 / 6$ weekly yoga classes were not included in the analysis. All analyses were conducted using IBM-SPSS v.25. Individual changes in pain and other symptoms were assessed using Students' paired samples $t$-test or Wilcoxon signed-ranks tests and Pearson or Spearman correlations were calculated to assess relationships between variables, as appropriate. In the group of participants who completed the study, the amount of missing data was relatively low for daily diary collection (5\%), with no missing preintervention and postintervention assessment data. Multilevel modeling was used in order to assess patient characteristics that were associated with better outcomes at the end of the yoga program (sixth week). Baseline to post-treatment percentage change was calculated as follows: $\{[$ Post/Pre $\times 100]-100\}$. Repeated measures of daily pain, fatigue, and sleep efficiency (Level 1 units) were nested within participants, and related to patients' baseline psychosocial characteristics (Level 2 units), including baseline anxiety (PROMIS-anxiety short form) and catastrophizing (PCS). Given that multilevel modeling can account for an unbalanced data structure and/or random missing data, ${ }^{27,28}$ no data imputation procedure was used. Two-way (e.g., Level 2 baseline catastrophizing $\times$ Level 2 baseline PROMIS-anxiety) interaction terms were also specified and included in the model, and baseline sleep efficiency, fatigue, 
and pain were included as covariates. We examined the amount of missing data for each of the main outcome variables (e.g. pain catastrophizing, pain intensity, daily fatigue, daily pain, FM symptoms, sleep quality). For the primary variables sleep quality, daily fatigue, pain severity, pain catastrophizing, and FM symptoms, there were no missing data. Non-completers were not included in the analysis, due to a lack of endpoint comparator for pre-post comparisons, and lack of 6-week data for multilevel modeling.

\section{Results}

Forty-six participants initially enrolled, and 36 participants completed the study. Participants who dropped out of the study ( $\mathrm{n}=10 ; 5$ at week 1, 1 at week 2, 1 at week 4 , and 3 at week 5) (see flow diagram, Appendix), most commonly cited lack of time to attend classes as the reason for noncontinuance.

\section{Demographic and baseline characteristics of sample (Table I)}

The average age of participants was $48.5 \pm 13.9$, all participants were women, and $83 \%$ were identified as Caucasian. The reported average pain severity, sleep disturbance, and psychosocial variables were similar to previous studies among patients with FM. ${ }^{29-31}$ Of those who initially agreed to participate, $74 \%(\mathrm{~N}=36)$ participated to a meaningful extent (attending 4-6 classes and reporting minutes of at home practice/day).

\section{Changes in pain}

Pain, as measured by the BPI, decreased from baseline to 6 weeks amongst the participants $(5.0 \pm 2.1$ vs $4.5 \pm 2.3$, $p=0.041$ ) and the highest reported daily pain (measured by daily diaries during baseline and week 6) decreased as well $(67 \pm 16$ vs $60 \pm 18, p=0.007)$ although substantial variability in pain scores was reported across participants (Figure 1A). Similarly, participants reported a variable amount of daily home practice, with more average home practice time associated with reduction in average pain (Spearman Rho: $-0.428, p=0.009$, Figure 1B). The greatest benefit was observed in those who practiced $25 \mathrm{mins} /$ day or more (28\% of subjects), compared to those who practiced less than $25 \mathrm{mins} /$ day $(p=0.023)$.

\section{Changes in FM symptoms: FIQR, fatigue, and sleep efficiency}

In contrast to the decrease in BPI, we did not observe a decrease in Revised FIQR group means (57.20 20.52 vs
Table I Demographics and baseline clinical characteristics

\begin{tabular}{|c|c|}
\hline Age & $48.5 \pm 13.9$ \\
\hline BMI & $28.2 \pm 7.1$ \\
\hline \multicolumn{2}{|l|}{ Race } \\
\hline Caucasian & $83 \%$ \\
\hline African American & $8 \%$ \\
\hline \multicolumn{2}{|l|}{ Education } \\
\hline Non-college graduate & $26 \%$ \\
\hline College graduate & $49 \%$ \\
\hline Master's degree & $20 \%$ \\
\hline Doctoral degree & $6 \%$ \\
\hline \multicolumn{2}{|l|}{ Employment } \\
\hline Full-time & $15 \%$ \\
\hline Part-time & $18 \%$ \\
\hline Homemaker & $23 \%$ \\
\hline Retired & $15 \%$ \\
\hline Daily average pain $(0-100)$ & $51.6 \pm 18.1$ \\
\hline Fybromyalgia symptoms (FIQR) & $57.2 \pm 20.5$ \\
\hline Pain (BPI) & $5.0 \pm 2.1$ \\
\hline Sleep disturbance (Pittsburgh Sleep Quality Index) & $11.3 \pm 4.5$ \\
\hline Daily fatigue $(0-100)$ & $60.7 \pm 17.5$ \\
\hline Catastrophizing (PCS) & $21.5 \pm 14.0$ \\
\hline Anxiety (PROMIS t-score) & $59.4 \pm 8.7$ \\
\hline
\end{tabular}

Abbreviations: BMI, body mass index; FIQR, Fibromyalgia Impact Questionnaire; BPI, Brief Pain Inventory; PCS, Pain Catastrophizing Scale; PROMIS, patientreported outcomes measurement system.

$53.18 \pm 18.12, p=0.44 ; 7 \%$ change). Because sleep disturbance is a prominent facet of FM symptomatology, we examined whether yoga-based exercise impacted sleep. Similar to pain, we observed a small overall decrease in sleep disturbance group mean on the PSQI (Figure 2A, $11.3 \pm 4.5$ vs $10.0 \pm 4.40$ $p=0.027,12 \%$ change) as well as fatigue (Figure $2 \mathrm{~B}, 60.7$ \pm 17.5 vs $54.0 \pm 17.8, p=0.003 ; 11 \%$ change), although this was quite variable between individuals. No significant change in sleep efficiency was detected as measured by actigraphy (Figure 2C, $87.1 \pm 7.6$ vs $85.5 \pm 9.9, p=0.59$, Wilcoxon signed rank), again with variability between individuals. However, a higher sleep efficiency at the end of the study (week 6 average sleep efficiency) was correlated with greater decreases in sleep disturbance over the course of the study (PSQI) (Rho: -0.40 , $p=0.03$ ), greater decreases in fatigue (rho: $-0.395, p=0.034$ ), and greater increases in overall self-reported physical activity (Rho: 0.395, $p=0.034$ ). 

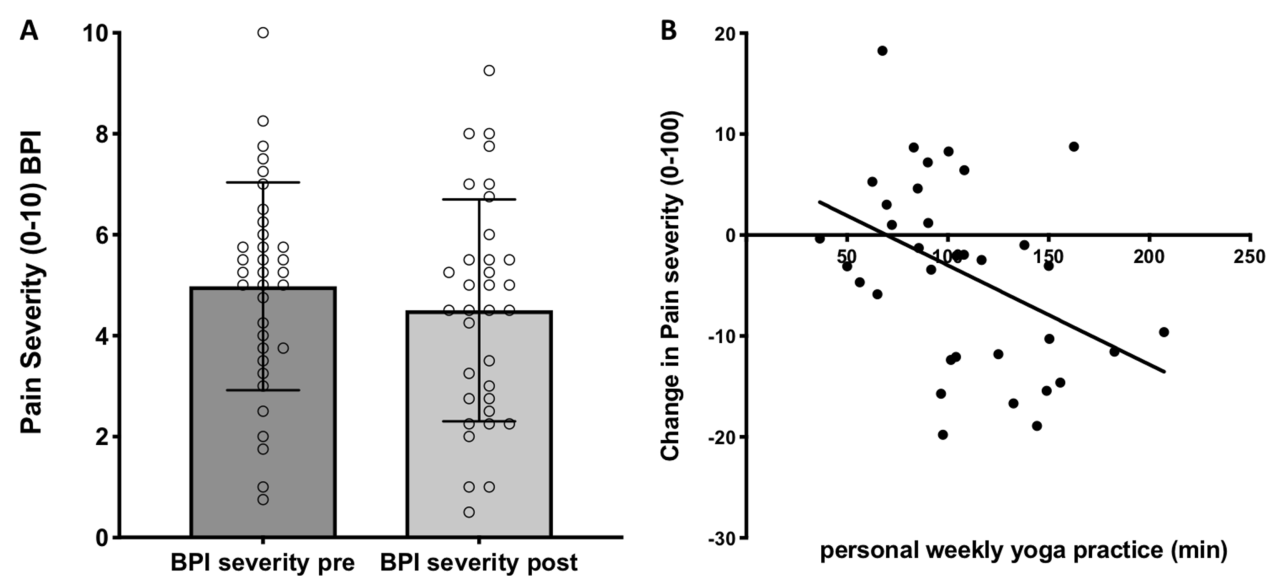

Figure I Changes in pain: association with amount of yoga practiced. (A) Pain intensity scores as measured using the brief pain inventory, either at baseline before (left) or after (right) yoga program; (B) correlation of decrease in average daily pain from baseline to 6th week of program with average reported minutes of home yoga practice over a 5 -day period.

A

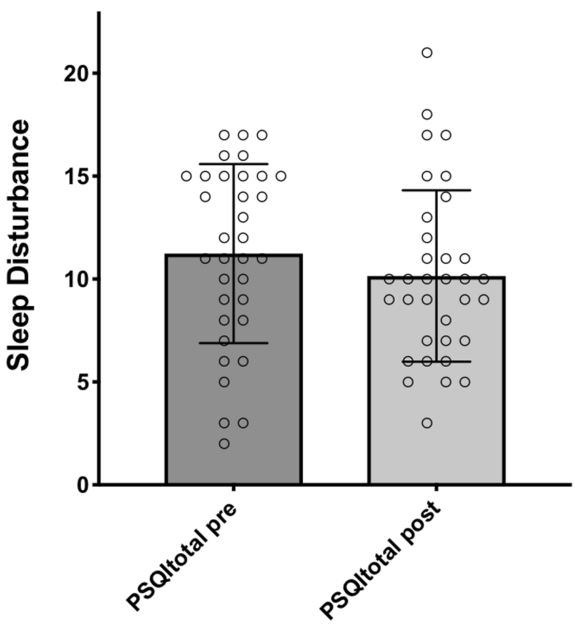

B

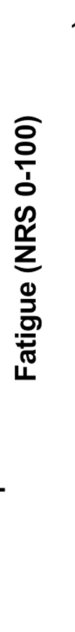

C

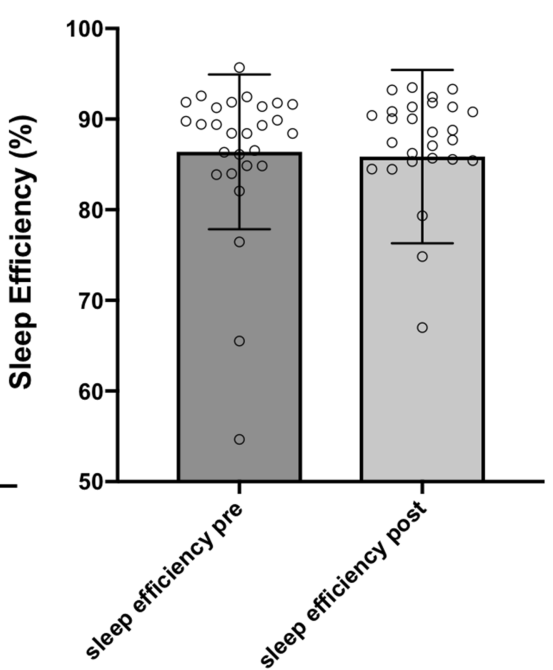

Figure 2 Changes in sleep and fatigue. (A) Sleep quality as measured by the pittsburgh sleep quality index, either at baseline before (left) or after (right) yoga program; (B) average fatigue $(0-100)$ measured by daily diaries, either at baseline (left) or during the 6th week of the yoga program (right); (C) sleep efficiency as measured using actigraphy, at baseline (left), and at end (right) of yoga program.

\section{Changes in pyschosocial functioning: pain catastrophizing}

Changes in pain catastrophizing were also variable amongst participants, with an overall decrease over the course of the program $(21.5 \pm 14$ vs $18.1 \pm 15, p=0.039$, $16 \%$ change Wilcoxon signed rank; Figure $3 \mathrm{~A}$ ). On the other hand, general anxiety, as measured by the PROMISanxiety short form, did not change $(59.38 \pm 8.7$ vs 60.19 $\pm 10.14 p<0.001)$.

Interestingly, those who showed a greater decrease in pain catastrophizing over the course of the program also reported a greater decrease in FM symptoms (FIQR) (Rho: 0.450, $p=0.006$, Figure 3B) as well as lower reported daily "worst" pain (Rho: 0.351, $p=0.036$ ). There was a trend toward a negative correlation between decreased catastrophizing and improved sleep efficiency (Rho: $-0.31, p=0.102$ ), but not between decreased anxiety and improved sleep efficiency (Rho: 0.09, $p=0.649$ ).

\section{Baseline characteristics predicting} symptoms at the end of yoga intervention In order to understand whether individual differences in psychosocial characteristics, such as catastrophizing and anxiety, could help explain variation in the degree of yoga's effect between individuals, we conducted multilevel modeling (MLM) analysis of pain and sleep. 

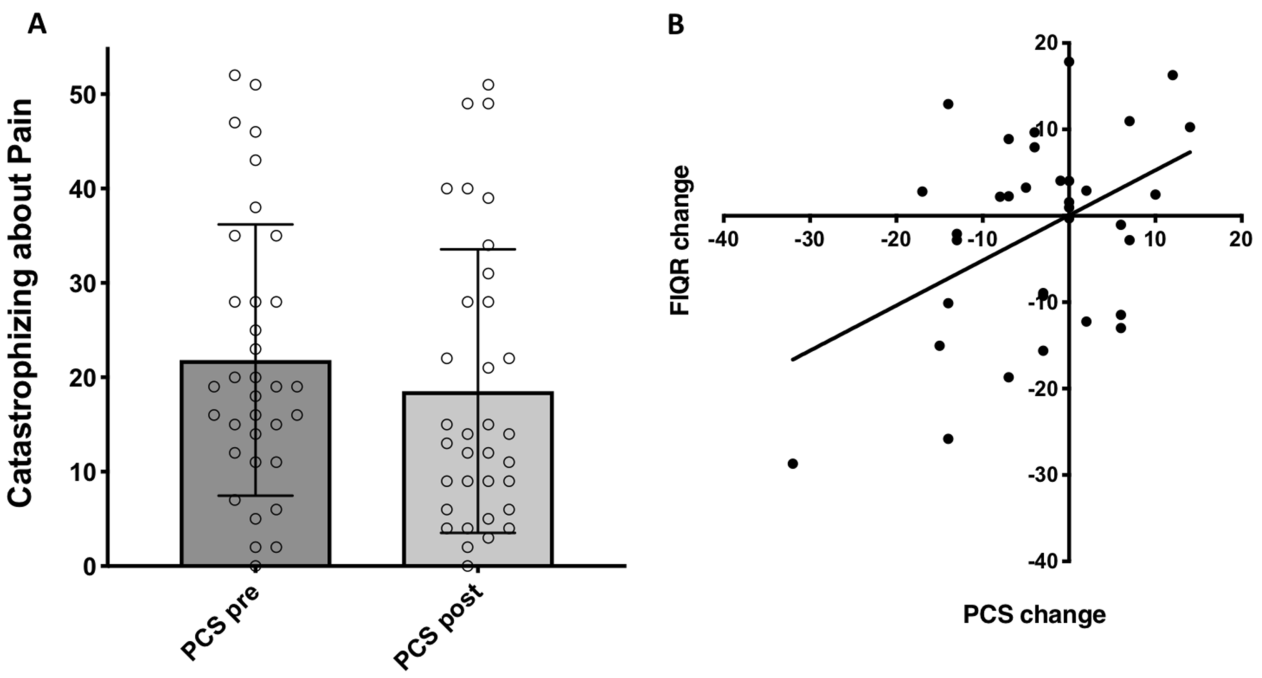

Figure 3 Changes in pain catastrophizing: association with changes in fibromyalgia symptoms. (A) Pain catastrophizing scores as measured using the pain catastrophizing scale (PCS), either at baseline before (left) or after (right) yoga program. (B) Correlation of decrease in fibromyalgia symptoms, as measured by change in pre- and postscores on the Revised Fibromyalgia Impact Questionnaire (FIQR), with decrease in pain catastrophizing.

Specifically, we used this method to investigate the influence of baseline catastrophizing and anxiety on the daily diary-rated pain and fatigue, as well as on actigraphymeasured sleep efficiency, during the final week of the yoga program, because FM symptoms (such as pain and sleep disturbance) vary considerably from day to day.

The results from the MLM analyses did not reveal a significant main effect of baseline pain catastrophizing score
(PCS) on sleep efficiency $(\mathrm{B}=0.041 \mathrm{SE}=0.16, p=0.80$ ), fatigue $(\mathrm{B}=0.059 \mathrm{SE}=0.28, p=0.83)$, or pain $(\mathrm{B}=-0.17 \mathrm{SE}=0.29$, $p=0.55)$. Similarly, there was no significant main effect of PROMIS-anxiety scores on sleep efficiency $(B=-0.077$ $\mathrm{SE}=0.26, p=0.77)$, fatigue $(\mathrm{B}=0.41, \mathrm{SE}=0.43, p=0.34)$, or pain $(\mathrm{B}=0.399, \mathrm{SE}=0.40, p=0.33)$. However, we observed a significant interaction between baseline PROMIS-anxiety and catastrophizing on post-study sleep efficiency $(B=-0.40$,
A

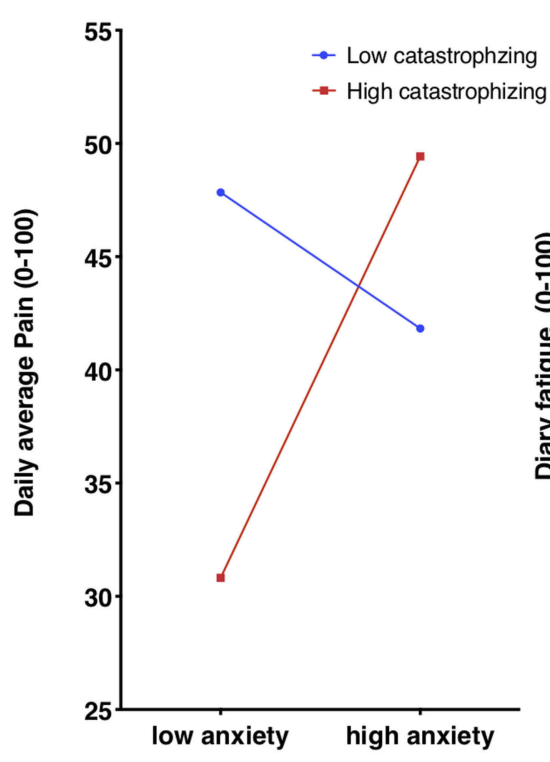

B

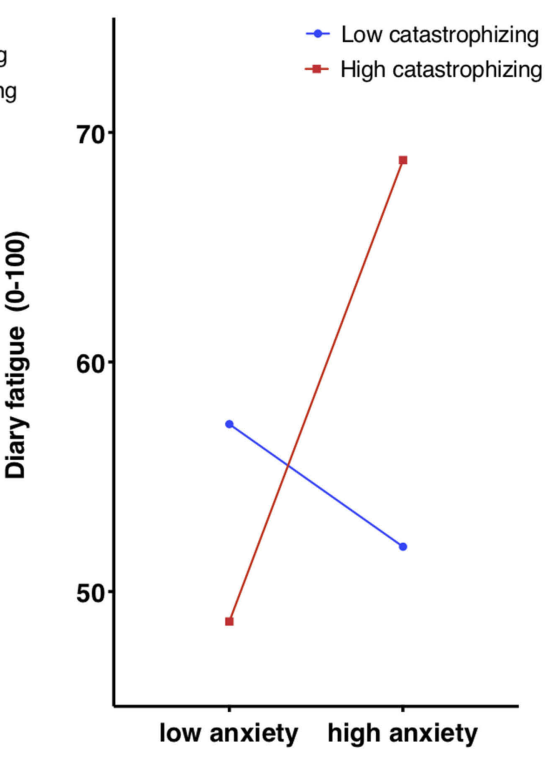

C

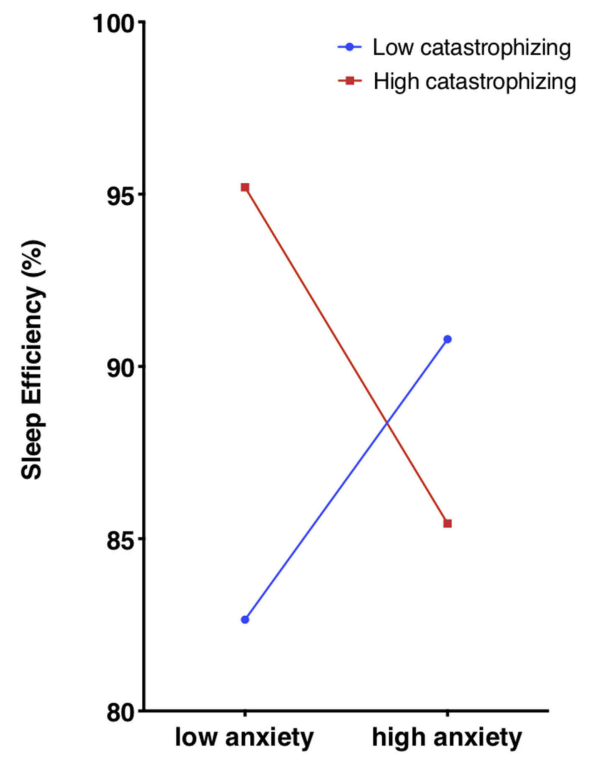

Figure 4 Pain, fatigue, and sleep efficiency at study end: association with baseline psychosocial characteristics. (A) Daily diary pain severity, (B) daily diary fatigue, and (C) nightly sleep efficiency (actigraphy) during the last week of yoga (week 6) was investigated using multilevel modeling to understand the impact of patients' baseline psychosocial characteristics. An interaction between baseline anxiety, as measured using PROMIS-anxiety, and baseline catastrophizing, as measured using the pain catastrophizing scale, was observed for all three outcomes. 
$\mathrm{SE}=0.01, p=0.016$ ), fatigue $(\mathrm{B}=0.06 \mathrm{SE}=0.022, p=0.011)$, and pain $(\mathrm{B}=0.055 \mathrm{SE}=0.02, p=0.011$ ), such that patients who scored high in catastrophizing and low in anxiety demonstrated significantly less fatigue, and pain, and higher sleep efficiency (Figure 4), after controlling for baseline fatigue, sleep efficiency and pain severity. In addition, sensitivity analyses indicated that the interaction was not moderated by any other demographic (i.e., age, sex, ethnicity) or psychological (i.e., anxiety, depression) variables (all $p$ 's $>0.05$ ).

\section{Discussion}

This pilot study in FM patients suggests that an exercisebased Satyananda yoga intervention may modulate some of the core symptoms of FM, including pain, sleep disturbance, and catastrophizing, but that this benefit is quite variable amongst individuals, resulting in meaningful benefit only to some. Exploratory analysis demonstrated that benefit was more pronounced for individuals who practiced more consistently, and who reported higher baseline catastrophizing, but low anxiety, in terms of sleep efficiency, fatigue, and pain at the end of the program.

The present finding that pain modestly decreased from baseline to postintervention for the overall group parallels results from previous yoga and meditation-based research interventions in chronic pain populations. ${ }^{9,32,33}$ These studies have shown a modest effect on reducing pain and disability, pain catastrophizing and acceptance, as well as cortisol, proinflammatory cytokines, and endorphin levels. ${ }^{1,7-14,34-36}$

Further analysis revealed the degree of benefit to be unequally distributed among participants, with some participants reporting more marked pain reduction. Specifically, pain reduction was inversely correlated with a greater amount of home practice, and most pronounced in those reporting greater than 25 mins of home practice on average. This suggests that greater engagement and participation may yield benefits in terms of pain reduction, which is in agreement with prior studies. ${ }^{37}$ Use of daily yoga videos at home, which featured the same instructor, may have assisted with practice adherence and engagement, but further investigation is required to determine barriers and facilitators to self-practice.

Laboratory-based, clinical, and epidemiological studies have suggested that sleep duration and quality influence subsequent pain report. Sleep deprivation in healthy subjects results in enhanced pain, and poor sleep is correlated with elevated pain severity and disability in individuals with chronic pain. ${ }^{11,38}$ Patients with sleep disturbance are more likely to develop chronic pain, and degree of sleep disturbance predicts severity of FM symptoms. ${ }^{12,13}$ Epidemiologic surveys consistently report that the vast majority of patients with FM suffer from significant disturbances initiating or maintaining sleep, ${ }^{15}$ and robust relationships are observed between pain severity and sleep disturbance in FM patients. ${ }^{16}$ Our study indicated beneficial effects of yoga practice on sleep quality and subjective fatigue in patients with FM, similar to earlier studies. ${ }^{8,39-41}$ Yoga appears to decrease autonomic reactivity, suggesting a fundamental normalization of physical processes, ${ }^{42}$ which may favor a transition to more normal sleep. More efficient sleep at the end of the study (based on wrist actigraphy) mirrored subjective sleep quality improvement and was associated with greater physical activity.

Patients with high catastrophizing and low anxiety at the beginning of the study had the most favorable sleep efficiency, reported better sleep, and less pain during the final week of Satyananda yoga. This interaction between catastrophizing and anxiety with respect to pain and sleep quality may indicate that participants who engage in negative thinking about symptoms (i.e. catastrophizing) might stand the most to gain from both the physical exercises and meditative practices inherent in yoga, but only if they have relatively low generalized anxiety. Previous studies have indicated that yoga allows patients to overcome the fear of movement, ${ }^{43}$ which is similar conceptually to catastrophizing about pain. Catastrophizing, which is higher amongst individuals with FM, was modestly decreased overall in the group during this study. Interestingly, we did also observe that decreases in reported FM symptoms (FIQR) during the course of the yoga program were associated with decreases in catastrophizing, perhaps indicating that FM symptom reduction may be connected to decreased catastrophizing in FM patients. ${ }^{3,44}$ However, a higher level of general anxiety might preclude effective engagement with yoga practice. Although prior studies have demonstrated that yoga helps decrease anxiety, this might be only the case in individuals with mild-moderate levels of anxiety at preintervention. ${ }^{45}$

The most important limitation of this pilot study was a relatively small sample size without a control group, making the generalizability of positive findings, as well as the definitiveness of negative findings, unclear. While we observed trends toward change in many variables, some did not reach statistical significance, raising the possibility that the study was underpowered for these outcomes. 
Second, as is apparent in the figures, the between subject variability was high, also limiting statistical power. We were able to partially mitigate the impact of variability by using paired tests and MLM analysis of daily diary and actigraphy data. However, it is likely that the degree of variation between individuals reflects a true variability in the experience of symptoms between individuals with FM, with some people gaining more benefit (or less) from the practice of yoga. Third, because of the voluntary nature of the intervention, substantial selection bias may exist, limiting the application of findings to individuals who are willing and able to engage in yoga. Fourth, approximately a quarter of initially interested individuals dropped out of meaningful participation, suggesting that this type of intervention may be too cumbersome for a significant subset of FM patients, and precluding our ability to assess its benefit to FM patients more generally. Fifth, this particular yoga intervention included gentle postures, anchored by a short meditation, and included daily at home video-led practice. It is unclear which of these elements was helpful/not helpful, and to what degree this may generalize to other yoga, exercise, or meditation practices. Further evaluation in a larger sample of patients, with a time-and-attention matched control would allow confirmation of the efficacy and tolerability of group-based yoga therapy for this challenging condition. The selection of meaningful active and passive control conditions will be important to understand whether and for whom yoga may be more useful.

\section{Conclusion}

This pilot study suggests that yoga-based exercise can be effective in decreasing pain, catastrophizing, and sleep disturbance in some FM patients, particularly those who are willing to engage in a more consistent home self-paced practice. As uptake of Satyananda yoga in FM can be variable, future research should potentially explore the efficacy of other yoga styles and protocols. Additionally, individual phenotypic characteristics that may predict the better clinical effect of yoga on fatigue, sleep efficiency, and pain include high catastrophizing, but relatively low general anxiety, although this should be investigated in a larger sample before being applied to clinical recommendation. Overall, yoga, as a safe, gentle, and adaptable exercise option, could be a beneficial tool to complement the conventional therapies currently used to manage FM symptoms.

\section{Acknowledgment}

The authors would like to sincerely thank all the participants who gave their time and energy to making this study possible. This study was supported by a grant to KLS from the Osher Center for Integrative Medicine/Harvard Medical School.

\section{Disclosure}

The authors report no conflicts of interest in this work.

\section{References}

1. Ward L, Stebbings S, Cherkin D, Baxter GD. Yoga for functional ability, pain and psychosocial outcomes in musculoskeletal conditions: a systematic review and meta-analysis. Musculoskeletal Care. 2013;11(4):203-217. doi:10.1002/msc. 1042

2. Schmidt-Wilcke T, Clauw DJ. Fibromyalgia: from pathophysiology to therapy. Nat Rev Rheumatol. 2011;7(9):518. doi:10.1038/ nrrheum.2011.2

3. Edwards RR, Cahalan C, Mensing G, Smith M, Haythornthwaite JA. Pain, catastrophizing, and depression in the rheumatic diseases. Nat Rev Rheumatol. 2011;7(4):216-224. doi:10.1038/nrrheum.2011.2

4. Schreiber KL, Campbell C, Martel MO, et al. Distraction analgesia in chronic pain patientsthe impact of catastrophizing. Anesthesiology. 2014;121(6):1292-1301. doi:10.1097/ALN.0000000000000465

5. Hauser W, Klose P, Langhorst J, et al. Efficacy of different types of aerobic exercise in fibromyalgia syndrome: a systematic review and meta-analysis of randomised controlled trials. Arthritis Res Ther. 2010;12(3):R79. doi:10.1186/ar3002

6. Busch AJ, Webber SC, Richards RS, et al. Resistance exercise training for fibromyalgia. Cochrane Database Syst Rev. 2013;12:Cd010884.

7. Carson JW, Carson KM, Jones KD, Bennett RM, Wright CL, Mist SD. A pilot randomized controlled trial of the yoga of awareness program in the management of fibromyalgia. Pain. 2010;151(2):530539. doi:10.1016/j.pain.2010.08.020

8. Carson JW, Carson KM, Jones KD, Mist SD, Bennett RM. Follow-up of yoga of awareness for fibromyalgia: results at 3 months and replication in the wait-list group. Clin J Pain. 2012;28(9):804-813. doi:10.1097/AJP.0b013e31824549b5

9. Tilbrook HE, Cox H, Hewitt CE, et al. Yoga for chronic low back pain: a randomized trial. Ann Intern Med. 2011;155(9):569-578. doi:10.7326/0003-4819-155-9-201111010-00003

10. Sherman KJ, Cherkin DC, Wellman RD, et al. A randomized trial comparing yoga, stretching, and a self-care book for chronic low back pain. Arch Intern Med. 2011;171(22):2019-2026. doi:10.1001/ archinternmed.2011.524

11. Fabian LA, McGuire L, Page GG, Goodin BR, Edwards RR, Haythornthwaite J. The association of the cortisol awakening response with experimental pain ratings. Psychoneuroendocrinology. 2009;34(8):1247-1251. doi:10.1016/j.psyneuen.2009.03.008

12. Ablin JN, Buskila D. Predicting fibromyalgia, a narrative review: are we better than fools and children? Eur J Pain. 2014;18(8):10601066. doi:10.1002/j.1532-2149.2014.00481.x

13. Finan PH, Goodin BR, Smith MT. The association of sleep and pain: an update and a path forward. J Pain. 2013;14(12):1539-1552. doi:10.1016/j.jpain.2013.08.007

14. Schreiber K, Loggia M, Cahalan C, Napadow V, Edwards R. (211) Modulation of experimental and clinical pain by distraction in fibromyalgia patients and controls. J Pain. 2015;16(4):S28. doi:10.1016/j. jpain.2015.01.126

15. Clauw DJ. Fibromyalgia: a clinical review. Jama. 2014;311 (15):1547-1555. doi:10.1001/jama.2014.3266 
16. Diaz-Piedra C, Di Stasi LL, Baldwin CM, Buela-Casal G, Catena A. Sleep disturbances of adult women suffering from fibromyalgia: a systematic review of observational studies. Sleep Med Rev. 2015;21:86-99. doi:10.1016/j.smrv.2014.09.001

17. Saraswati SS. Yoga Nidra. Yoga Publications Trust; 2009.

18. Saraswati SS. Asana Pranayama Mudra Bandha. Yoga Publications Trust; 2008.

19. Sullivan MJ, Bishop SR, Pivik J. The pain catastrophizing scale: development and validation. Psychol Assess. 1995;7(4):524-532. doi:10.1037/1040-3590.7.4.524

20. Pavlin DJ, Sullivan MJ, Freund PR, Roesen K. Catastrophizing: a risk factor for postsurgical pain. Clin J Pain. 2005;21(1):83-90.

21. Osman A, Barrios FX, Kopper BA, Hauptmann W, Jones J, O’Neill E. Factor structure, reliability, and validity of the pain catastrophizing scale. J Behav Med. 1997;20(6):589-605.

22. Pilkonis PA, Choi SW, Salsman JM, et al. Assessment of selfreported negative affect in the NIH toolbox. Psychiatry Res. 2013;206(1):88-97. doi:10.1016/j.psychres.2012.09.034

23. Cella D, Riley W, Stone A, et al. The Patient-Reported Outcomes Measurement Information System (PROMIS) developed and tested its first wave of adult self-reported health outcome item banks: 20052008. J Clin Epidemiol. 2010;63(11):1179-1194. doi:10.1016/j. jclinepi.2010.04.011

24. Bennett RM, Friend R, Jones KD, Ward R, Han BK, Ross RL. The revised Fibromyalgia Impact Questionnaire (FIQR): validation and psychometric properties. Arthritis Res Ther. 2009;11(4):R120. doi:10.1186/ar2783

25. Buysse DJ, Reynolds III CF, Monk TH, Berman SR, Kupfer DJ. The pittsburgh sleep quality index: a new instrument for psychiatric practice and research. Psychiatry Res. 1989;28(2):193-213. doi:10.1016/0165-1781(89)90047-4

26. Cleeland C, Ryan K. Pain assessment: global use of the brief pain inventory. Ann Acad Med. 1994;23(2):129-138.

27. Peugh JL. A practical guide to multilevel modeling. J Sch Psychol. 2010;48(1):85-112. doi:10.1016/j.jsp.2009.09.002

28. Singer JD, Willett JB. Applied Longitudinal Data Analysis: Modeling Change and Event Occurrence. Oxford;New York: Oxford University Press; 2003:xx, 644.

29. Brummett CM, Clauw DJ. Fibromyalgia: a primer for the anesthesia community. Curr Opin Anaesthesiol. 2011;24(5):532. doi:10.1097/ ACO.0b013e32834a1091

30. Clauw DJ, Arnold LM, McCarberg BH, editors. The science of fibromyalgia. In: Mayo Clinic Proceedings. Elsevier; 2011;86 (9):907-911.

31. Hawkins RA. Fibromyalgia: a clinical update. J Am Osteopath Assoc. 2013;113(9):680-689. doi:10.7556/jaoa.2013.034

32. Williams K, Abildso C, Steinberg L, et al. Evaluation of the effectiveness and efficacy of Iyengar yoga therapy on chronic low back pain. Spine (Phila Pa 1976). 2009;34(19):2066-2076. doi:10.1097/ BRS.0b013e3181b315cc
33. Tiedemann A, O'Rourke S, Sherrington C. Is a yoga-based program with potential to decrease falls perceived to be acceptable to community-dwelling people older than 60? Public Health Res Pract. 2018;28(2):e28011801. doi:10.17061/phrp28011801

34. Curtis K, Osadchuk A, Katz J. An eight-week yoga intervention is associated with improvements in pain, psychological functioning and mindfulness, and changes in cortisol levels in women with fibromyalgia. J Pain Res. 2011;4:189-201. doi:10.2147/JPR. S22761

35. Michalsen A, Grossman P, Acil A, et al. Rapid stress reduction and anxiolysis among distressed women as a consequence of a threemonth intensive yoga program. Med Sci Monit. 2005;11(12):Cr555Cr561.

36. Yadav RK, Magan D, Mehta N, Sharma R, Mahapatra SC. Efficacy of a short-term yoga-based lifestyle intervention in reducing stress and inflammation: preliminary results. J Altern Complement Med. 2012;18(7):662-667. doi:10.1089/acm.2011.0265

37. Santana MJ, SP J, Mirus J, Loadman M, Lien DC, Feeny D. An assessment of the effects of Iyengar yoga practice on the healthrelated quality of life of patients with chronic respiratory diseases: a pilot study. Can Respir J. 2013;20(2):e17-e23. doi:10.1155/2013/ 562104

38. Cohen S, Kamarck T, Mermelstein R. A global measure of perceived stress. J Health Soc Behav. 1983;24(4):385-396.

39. Wang YY, Chang HY, Lin CY. [Systematic review of yoga for depression and quality of sleep in the elderly]. $\mathrm{Hu} \mathrm{Li} \mathrm{Za} \mathrm{Zhi.}$ 2014;61(1):85-92.

40. Wang F, Eun-Kyoung Lee O, Feng F, et al. The effect of meditative movement on sleep quality: a systematic review. Sleep Med Rev. 2016;30:43-52. doi:10.1016/j.smrv.2015.12.001

41. Carson JW, Carson KM, Jones KD, Lancaster L, Mist SD. Mindful yoga pilot study shows modulation of abnormal pain processing in fibromyalgia patients. Int $J$ Yoga Therap. 2016;26(1):93-100. doi:10.17761/1531-2054-26.1.93

42. Kanojia S, Sharma VK, Gandhi A, Kapoor R, Kukreja A, Subramanian SK. Effect of yoga on autonomic functions and psychological status during both phases of menstrual cycle in young healthy females. J Clin Diagn Res. 2013;7(10):2133.

43. Combs MA, Thorn BE. Yoga attitudes in chronic low back pain: roles of catastrophizing and fear of movement. Complement Ther Clin Pract. 2015;21(3):160-165. doi:10.1016/j.ctcp.2015.06.006

44. Campbell CM, McCauley L, Bounds SC, et al. Changes in pain catastrophizing predict later changes in fibromyalgia clinical and experimental pain report: cross-lagged panel analyses of dispositional and situational catastrophizing. Arthritis Res Ther. 2012;14(5):R231. doi:10.1186/ar4073

45. Wieland LS, Santesso N. A summary of a cochrane review: yoga treatment for chronic non-specific low back pain. Eur J Integr Med. 2017;11:39-40. doi:10.1016/j.eujim.2017.03.003
Journal of Pain Research

\section{Publish your work in this journal}

The Journal of Pain Research is an international, peer reviewed, open access, online journal that welcomes laboratory and clinical findings in the fields of pain research and the prevention and management of pain. Original research, reviews, symposium reports, hypothesis formation and commentaries are all considered for publication. The manuscript

Submit your manuscript here: https://www.dovepress.com/journal-of-pain-research-journal management system is completely online and includes a very quick and fair peer-review system, which is all easy to use. Visit http:// www.dovepress.com/testimonials.php to read real quotes from published authors. 